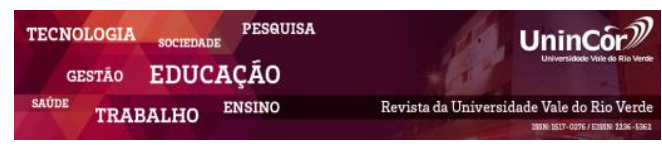

Revista da Universidade Vale do Rio Verde ISSN: 1517-0276 / EISSN: 2236-5362 Vol. 16 | n. 1 | Ano 2018

Rafael Pereira

Universidade Federal do Ceará rafaelpereirabiologo@gmail.com

Mariana Ferreira do Nascimento Universidade Estadual Vale do Acaraú maryanna_lindda@hotmail.com

Danielle Rodrigues Maciel

Universidade Estadual Vale do Acaraú daniellekatunda@hotmail.com

Murilo Sérgio da Silva Julião Universidade Estadual Vale do Acaraú murilo-sergio@uol.com.br

Hélcio Silva Santos Universidade Estadual Vale do Acaraú helciodossantos@gmail.com

Selene Maia de Morais Universidade Estadual do Ceará selenemaiademorais@gmail.com

Raquel Oliveira dos Santos Fontenelle Universidade Estadual Vale do Acaraú raquelbios@yahoo.com.br

\section{ABORDAGEM FITOQUÍMICA DO EXTRATO ETANÓLICO DA CASCA DE Myroxylon peruiferum $\mathrm{L.f}$}

\begin{abstract}
RESUMO
O uso de produtos naturais com propriedades terapêuticas é tão antigo quanto à civilização humana e, por um longo período, minerais, plantas e produtos animais eram fundamentais fontes de drogas. Myroxylon peruiferum L.f. pertence à família Fabaceae e é conhecida popularmente como bálsamo. O objetivo deste trabalho foi avaliar a prospecção fitoquímica do extrato etanólico da casca de $M$. peruiferum L.f. As propriedades fitoquímica do extrato foram determinadas pelos métodos padrões descritas por Matos. A determinação da atividade antioxidante seguiu o método de varredura do radical livre DPPH. Para a quantificação de flavonoides, seguiu-se a metodologia proposta por Funari e Ferro. Os resultados demonstram que na casca do bálsamo há uma ampla disponibilidade de ferro e de ácido ascórbico. O extrato apresenta taninos, flavonoides, triterpenoides e saponinas. Em relação ao DPPH, o extrato etanólico apresenta boa capacidade antioxidante frente ao radical. Os resultados demonstram o potencial químico, assim como justificam a sua utilização na medicina popular para o tratamento de doenças.
\end{abstract}

Palavras-chave: Medicina popular. Myroxylon peruiferum L.f. Prospecção. Produtos Naturais. DPPH.

\section{PHYCHOCHEMICAL APPROACH OF THE ETHANOLIC EXTRACT OF THE Myroxylon peruiferum L.f}

\begin{abstract}
The use of natural products with therapeutic properties is as old as the human civilization and for a long period, mineral, plant and animal products were essential sources of drugs. Myroxylon peruiferum $L$. f. belongs to Fabaceae family and is popularly known as balm. This study aimed to evaluate the phytochemical properties of the ethanol extract of M. peruiferum L.f. bark. The phytochemical properties of the extract were determined by standard methods described by Matos. The determination of antioxidant activity was based on the free radical DPPH scanning method. For quantification of flavonoids, followed the methodology proposed by Funari and Ferro. The results demonstrate that the balsam shell contains a wide availability of iron and ascorbic acid (vitamin C). The extract has tannins, flavonoids, triterpenoids and saponins. Regarding the DPPH, the ethanol extract showed a good antioxidant capacity against radical. The results demonstrate the chemical potential, as well as justify their use in folk medicine for the treatment of diseases.
\end{abstract}

Keywords: Medicine popular. Myroxylon peruiferum L.f. Prospection. Natural products. DPPH.. 


\section{INTRODUÇÃO}

$\mathrm{O}$ uso de produtos naturais com propriedades terapêuticas é tão antigo quanto à civilização humana e, por um longo período, minerais, plantas e produtos animais eram fundamentais fontes de drogas (BAJPAI et al., 2008). A ampla biodiversidade de espécies vegetais, existentes no Brasil, designa uma de suas maiores riquezas que se destacam como motivo para a obtenção de novas substâncias para utilidade terapêutica (KORDALI et al., 2008). Plantas, por exemplo, são bastante usadas na medicação de diversas enfermidades, infecciosas ou não (MACIEL; VIANA, 2005).

Embora haja aumento nos estudos sobre plantas medicinais, apenas de $15 \%$ a $17 \%$ do seu potencial medicinal foram estudados. Em relação à imensa biodiversidade do Nordeste brasileiro, esse número poderia ser bem maior (ALMEIDA et al., 2006). No Ceará, mais especificamente, várias pesquisas vêm sendo desenvolvidas com a finalidade de confirmação científica dos produtos naturais extraídos de plantas, que são utilizadas pela medicina tradicional, dentre os quais, podemos citar os trabalhos de Lahlou et al. (2000), Bertini et al. (2005), Morais et al. (2006), Almeida et al. (2006), Botelho et al. (2007), Fontenelle et al. (2007 e 2008) e Soares et al. (2015).

Myroxylon peruiferum L.f. pertence à família Fabaceae (antiga Leguminosae e Papilionoideae), conhecida popularmente como cabriúva, pau-de-bálsamo e bálsamo. É uma planta decídua, heliófita, possuindo cerca de 10 a 20 metros de altura. Suas folhas são compostas pinadas, de ocorrência em quase toda a extensão do País e a madeira é adequada para mobiliário, revestimentos, construção civil, dentre outras atribuições, por causa da sua alta resistência ao apodrecimento (LORENZI, 1998).

Diante do exposto, o objetivo deste trabalho foi avaliar a abordagem fitoquímica do extrato etanólico da casca de Myroxylon peruiferum L. f.

\section{MÉTODOS}

Material vegetal e preparação do extrato

Casca de Myroxylon peruiferum L.f. foi coletada no maciço de Uruburetama, localizado no distrito de Soledade no município de Itapajé, sob as coordenadas $03^{\circ} 37^{\prime} 86^{\prime}$ 'S e $39^{\circ} 35^{\prime} 33^{\prime}$ ' W, com altitude de $800 \mathrm{~m}$. A exsicata da planta encontra-se arquivada no Herbário Prof. Francisco José de Abreu Matos da Universidade Estadual Vale do Acaraú, autenticada pelo Dr. E. B. Souza sob o tombo 19240.

As cascas foram secas à temperatura ambiente e trituradas utilizando um moinho. Posteriormente, o material foi armazenado em recipiente com etanol por 4 dias. O extrato foi evaporado a vácuo usando o rotaevaporador. 
Separação das frações

O extrato etanólico bruto (100g), depois de concentrado a vácuo, foi cromatografado em coluna de sílica em gel com eluentes de polaridades crescente (hexano, diclorometano, clorofórmio, acetato de ética, metanol e etanol). As soluções foram novamente rotaevaporada para obter as frações do extrato bruto.

\section{Constituintes minerais}

Para a determinação de cálcio foi utilizado o método gravimétrico. $\mathrm{O}$ método iodométrico foi utilizado para quantificar o ácido ascórbico (vitamina C). Para a determinação do ferro foi aplicada a metodologia de permanganometria. Já para o potássio foi determinada por fotometria de chama. (AOAC, 2012). Todas as determinações foram realizadas em triplicata.

\section{Análise fitoquímica}

As propriedades fitoquímica do extrato foram determinadas pelos métodos padrões descritas por Matos (1988). A determinação foi para taninos, flavonoides, esteroides, triterpenoides, saponinas e alcaloides.

Quantificação de flavonoides e fenóis totais

Para a quantificação de flavonoides, seguiu-se a metodologia proposta por Funari e Ferro (2006). Este teste foi realizado em triplicata. A quantificação do teor de fenóis totais foi feita por meio de espectrofotômetro tendo comprimento de onda de $750 \mathrm{~nm}$ e utilizando o reagente Folin-Ciocalteu e a curva padrão do ácido gálico (SOUSA et al., 2007).
Atividade antioxidante pelo método-sequestrador de radicais livres DPPH

A determinação da atividade antioxidante seguiu o método sequestrador de radicais livres DPPH (1,1-difenil-2-picril-hidrazila), descrito por Yepez et al. (2002). Em um tubo de ensaio, foram colocados $3,9 \mathrm{~mL}$ da solução metanólica $6,5 \times 10^{-}$ ${ }^{5} \mathrm{M}$ do radical livre DPPH. Depois disso, foi adicionado no tubo $0,1 \mathrm{~mL}$ da solução metanólica da amostra que foram testadas nas concentrações de $250 \mathrm{mg} / \mathrm{mL}, 125 \mathrm{mg} / \mathrm{mL}, 25 \mathrm{mg} / \mathrm{mL}, 12,5$ $\mathrm{mg} / \mathrm{mL}, 2,5 \mathrm{mg} / \mathrm{mL}, 1,25 \mathrm{mg} / \mathrm{mL}, 0,25 \mathrm{mg} / \mathrm{mL}$, $0,125 \mathrm{mg} / \mathrm{mL}$ e $0,025 \mathrm{mg} / \mathrm{mL}$. Após o período de 60 min foi conferida a absorbância num espectrofotômetro Spekol no comprimento de onde de $515 \mathrm{~nm}$. Os resultados foram utilizados para calcular o índice de varredura da amostra em percentual (IV\%), utilizando a formula: IV\%= $\left(\mathrm{A}_{\mathrm{DPPH}}-\mathrm{A}_{\mathrm{AMOSTRA}} / \mathrm{A}_{\mathrm{DPPH}}\right) \times 100$. Em que o IV\% é o índice de varredura do DPPH e o A foi o valor final da absorbância depois do período de repouso. Os números encontrados foram aplicados no Programa Estatístico Origin 7.0 para o cálculo da concentração que inibiu $50 \%$ dos radicais livres das amostras. O teste foi feito em triplicata para cada concentração. Para comparação, usou-se a quercetina como padrão na mesma concentração do extrato.

\section{RESULTADOS E DISCUSSÃO}

O maciço de Uruburetama está situado num dos maciços residuais do Ceará, que apresenta tipo de relevo montanhoso de formação cristalina. Talvez por consequência do alto grau 
de antropização, há pouco interesse em estudar a flora dessa região, porém estudos recentes demonstram que os remanescentes de Mata Atlântica da Serra apresentam uma considerável riqueza vegetal, mostrando a importância de novas pesquisas (MATOS NETO, 2010; LIMA, 2012).

Os resultados sobre a composição mineral e o desvio padrão do extrato estão sumarizados na tabela 1 e demostra que na casca de $M$. peruiferum L.f. há uma ampla disponibilidade de ferro e de ácido ascórbico (vitamina C).

Porém, foram encontrados baixos teores para cálcio e potássio. Isso constata que o extrato bruto e a fração metanólica foram os melhores solventes extratores em que apresentaram 4 grupos químicos dos 6 estudados.

Tabela 1 - Composição mineral e desvio padrão do extrato de M. peruiferum L.f.

\begin{tabular}{lr}
\hline Micronutriente & Valor $(\%) \pm$ SD \\
\hline Ácido ascórbico & $3,38 \pm 0,69$ \\
Cálcio & $1,59 \pm 0,78$ \\
Ferro & $2,43 \pm 0,06$ \\
Potássio & $0,39 \pm 0,05$ \\
\hline
\end{tabular}

Fonte: $\mathrm{O}$ autor.

Fundamenta-se que a partir dos extratos de distintas polaridades das plantas, foi possível observar que nos vegetais há presença de taninos, flavonoides, esteroides, saponinas e alcaloides, o que praticamente explica a atividade antimicrobiana (COELHO, 2003).

Tabela 2 - Composições fitoquímica das cascas do M. peruiferum L.f.

\begin{tabular}{lccc}
\hline Composição Fitoquímica & Bruto & Acetato & Metanol \\
\hline Taninos & + & + & + \\
Flavonoides & + & + & + \\
Esteroides & - & - & + \\
Triterpenoides & + & - & + \\
Saponinas & + & + & - \\
Alcaloides & - & - & \\
\hline
\end{tabular}

(+) Positivo; (-) negativo.

Fonte: $\mathrm{O}$ autor

A importância de se encontrar uma elevada concentração de ferro em extratos de plantas, mesmo sendo um micronutriente, é a sua utilização como aditivo alimentar no combate à anemia. Enquanto o ácido ascórbico poderá servir para evitar a avitaminose.

Com a realização da caracterização fitoquímica, foi possível observar grupos de 
compostos químicos derivados do metabolismo secundário das plantas, como taninos, flavonoides, triterpenoides e saponinas. A Tabela 2 mostra os resultados encontrados na triagem fitoquímica a partir do extrato bruto e de diferentes frações da planta analisada.

Os resultados da quantificação do teor de fenóis totais e flavonoides, e os valores de $\mathrm{CE}_{50}$ (concentração da amostra capaz de sequestrar $50 \%$ dos radicais DPPH), determinados para o extrato de $M$. peruiferum L. f. e para o controle positivo (quercetina) são mostrados na tabela 3 .

O resultado demonstra que o extrato etanólico apresenta boa capacidade antioxidante frente ao radical DPPH. Foi verificado que a amostra apresenta uma $\mathrm{CE}_{50}$ menor que o controle positivo quercetina, e quanto menor o valor do $\mathrm{CE}_{50}$, maior a capacidade antioxidante de uma amostra.

Tabela 3 - Atividade antioxidante, teor de fenóis totais e flavonoides em M. peruiferum L. $\mathrm{f}$

\begin{tabular}{lccc}
\hline Extrato & $\begin{array}{c}\text { Teor de compostos } \\
\text { Fenólicos }\end{array}$ & Teor de Flavonoides & $\mathrm{CE}_{50}$ \\
\hline $\boldsymbol{E E C M P}$ & $28,72 \% \pm 2,31$ & $1,28 \% \pm 0,08$ & $0,056 \pm 1,09$
\end{tabular}

$\mathrm{CE}_{50}$, concentração da amostra que foi capaz de sequestrar 50\% dos radicais DPPH. EECMP: Extrato etanólico da casca de $M$. peruiferum L. f.

Fonte: $\mathrm{O}$ autor.

\section{CONCLUSÃO}

pois a espécie estudada mostra-se com relevantes atividades biológicas e químicas.

\section{REFERÊNCIAS}

Nesse estudo, podemos constatar que o extrato bruto assim como as frações acetato e metanólica de $M$. peruiferum L. f. apresentam grupos de compostos químicos derivados do metabolismo secundário das plantas. Contêm, ainda, elevados teores de ferro e de vitamina C, mostrando-se uma ampla variedade de substâncias farmacológicas ativas que podem estar relacionadas com todas as atividades biológicas. Em relação à atividade antioxidante, o extrato apresenta boa atividade, possivelmente atribuída aos componentes de classe fenólica presentes.

Neste contexto, a planta mostra-se como uma fonte promissora de matéria-prima na produção de novos medicamentos fitoterápicos,

ALMEIDA, C. F. C. B. R.; AMORIM, E. L. C.; ALBUQUERQUE, U. P.; MAIA, M. B. Medical plants popularly used in the Xingó region - a semiarid location in Northeastern Brazil. Journal Ethnobiol Ethnomed. v. 15, p. 1-7, 2006.

AOAC INTERNATIONAL. The Official Methods of Analysis. $19^{\circ} \mathrm{ed} .2012$.

BAJPAI, V. K.; SHUKLA, S.; KANG, S. C. Chemical composition and antifungal activity of essential oil and various extract of Silene armeria L. Bioresource

Technology. v.99, n.18,p. 8903-8908,2008.

BARBEDO, L. S.; SGARBI, D. B. G. Candidíase. Jornal brasileiro de Doenças Sexualmente Transmissíveis, Rio de Janeiro, v. 22, n.1, p. 22-38, 2010 .

BERTINI, L.M., PEREIRA, A.F., OLIVEIRA, C.L.L., MENEZES, E. A., MORAIS, S.M., CUNHA, F.A. CAVALCANTE, E.S.B. Perfil de sensibilidade de bactérias frente a óleos essenciais de algumas plantas do Nordeste do Brasil. Infarma, v. 17, p. 8083, 2005. 
COELHO, A. M. S. P. et al. Atividade antimicrobiana de Bixa orellana L. (Urucum). Revista Lecta. v. 21, n. 1/2, p. 47-54, 2003.

FONTENELLE, R. O. S.; MORAIS, S. M.; BRITO, E. H. S.; KERNTOPF, M. R.; BRILHANTE, R. S. N.; CORDEIRO, R. A.; TOMÉ, A. R.; QUEIROZ, M. G. R.; NASCIMENTO, N. R. F.; SIDRIM, J. J. C.; ROCHA, M. F. G. Chemical composition, toxicological aspects and antifungal activity of essential oil from Lippia sidoides Cham. J Antimicrob Chem.v.59, p. 934-940, 2007.

FONTENELLE, R. O. S.; MORAIS, S. M.; BRITO, É. H. S.; BRILHANTE R.S. N.; CORDEIRO, R. A.;NASCIMENTO, N.R. F.; MENDONÇA, M. R. K.; SIDRIM, J. J. C.; ROCHA, M. F. G. Antifungal activity of essential oils of Croton species from the Brazilian Caatinga biome. Journal of Applied Microbiology. v. 104, p. 1383-1390, 2008.

FUNARI, C.S; FERRO, V.O. Análise de própolis. Ciência e Tecnologia de Alimentos, v.26, n.1, p.171178, 2006.

KORDALI, S.; CAKIR, A.; OZER, H.; CAKMAKCI, R.; KESDEK, M.; METE, E. Antifungal, phytotoxic and insecticidal properties of essential oil isolated from Turkish Origanum acutidens and its three components, carvacrol, thymol and $p$-cymene. Bioresource Technology. v. 99,n.18, p.8788-8795, 2008.

LAHLOU, S.; LEAL-CARDOSO, J. H.; MAGALHÃES, P. J. Essential oil of Croton nepetaefolius decreases blood pressure through an action upon vascular smooth muscle: studies in DOCA-salt hypertensive rats. Planta Medica. v.66, n.2, p. 138-143, 2000.

LORENZI, H. Árvores Brasileiras: manual de identificação e cultivo de plantas arbóreas nativas do Brasil. Nova Odessa: Plantarun, 1998. 352p.

MACIEL, A. S.; VIANA, J. A. Dermatofitose em cães e gatos: uma revisão. Clínica Veterinária. v. 57, p. 74-82, 2005.

MATOS NETO, F. A. Abordagem etnobotânica de plantas medicinais usadas para tratamento de lesões de pele associado, ou não, a bactérias ou fungos no município de Itapajé, Ceará, Brasil.2013. 18f. Trabalho de conclusão de curso. Departamento de Biologia, Universidade Estadual Vale do Acaraú, Sobral, 2013.

MATOS NETO, F. A.; OLIVEIRA, H. C. Briófitas da Serra de Uruburetama, Ceará, Brasil. In: XII ENCONTRO DE INICIAÇÃO CIENTÍFICA DA UNIVERSIDADE ESTADUAL VALE DO ACARAÚ- UVA, 2010, Sobral. Anais do XII
Encontro de Iniciação Científica da Universidade Estadual Vale do Acaraú, 2010, p.2.

MATOS, F.J. A. Introdução à Fitoquímica Experimental. Fortaleza: UFC, 2009. 126p.

MORAIS, S. M.; CAVALCANTI, E. S. B.; BERTINI, L. M.; OLIVEIRA, C. L. L.; RODRIGUES, J. R. B.; CARDOSO, J. H. L. Larvicidal activity of essential oils from Brazilian Croton species against Aedes aegypti L. J Am Mosq. Control Assoc. v. 22, p. 161164, 2006.

SOARES, B. V.; MORAIS, S. M.; FONTENELLE, R. O. S.; BRITO É. H. S.; QUEIROZ, V. A.; VILANOVA, N. S.; CAVALCANTE, C. S. P.; ROCHA, M.F.G. Actividad antifúngica de extractos vegetales contra Microsporum canis y Candida spp. Las cepas. Boletín Latinoamericano y del Caribe de Plantas Medicinales y Aromáticas, v. 14, p. 251-262, 2015.

SOUSA, C.M.M.; SILVA H.R.; VIEIRA JÚNIOR, G.M. et al. Fenóis totais e atividade antioxidante de cinco plantas medicinais. Química Nova, v.30, n.2, p.351-355, 2007.

YEPEZ, B.;ESPINOSA, M.; LÓPEZ, S.; BOLAÑOS, G. Producing antioxidant fractions from herbaceous matrices by supercritical fluid extraction. Fluid Phase Equilibria. v. 194, p. 879-884, 2002.

Rafael Pereira
Mestre em Biotecnologia de Recursos Naturais-UFC.
Doutorando em Biotecnologia em Recursos Naturais-
UFC.

\section{Mariana Ferreira do Nascimento}

Discente do curso de química da Universidade Estadual Vale do Acaraú
Danielle Rodrigues Maciel

Discente do curso de química da Universidade Estadual Vale do Acaraú

\begin{tabular}{l}
\hline Murilo Sérgio da Silva Julião \\
Mestre e doutor em Química Analítica- USP \\
Professor associado da Universidade Estadual Vale \\
do Acaraú. \\
\hline \\
Hélcio Silva Santos \\
Mestre e doutor em Química Orgânica-UFC \\
Professor adjunto da Universidade Estadual Vale do \\
Acaraú.
\end{tabular}




\section{Selene Maia de Morais}

Mestra em Química Orgânica-UFC, doutora em Química-University of London.

Professora Titular da Universidade Estadual do Ceará

\section{Raquel Oliveira dos Santos Fontenelle}

Mestra e doutora em Ciências Veterinárias-UECE

Professora adjunta da Universidade Estadual Vale do

Acaraú. 